\title{
Boundary behaviour of the four-point function in the 3-dimensional Gross-Neveu model
}

\author{
A. P. C. Malbouisson* \\ Centre de Physique Théorique, Ecole Polytechnique 91128 Palaiseau Cedex, France \\ J. M. C. Malbouisson and A. E. Santana \\ Instituto de Física, Universidade Federal da Bahia, 40210-340, Salvador, BA, Brazil \\ J. C. da Silva \\ Centro Federal de Educação Tecnológica da Bahia, 40300-010, Salvador, BA, Brazil
}

\begin{abstract}
We consider the $N$-components 3-dimensional massive Gross-Neveu model compactified in one spatial direction, the system being constrained to a slab of thickness $L$. We derive a closed formula for the renormalized $L$-dependent four-point function at vanishing external momenta in the large- $N$ limit (the effective coupling constant), using bag-model boundary conditions. For values of the fixed coupling constant in absence of boundaries $\lambda \geq \lambda_{c} \simeq 19.16$, we obtain small-distance asymptotic freedom (for $L \rightarrow 0$ ) and a singularity for a length $L^{(c)}$ such that $2.07 \mathrm{~m}^{-1}<L^{(c)} \lesssim 2.82 \mathrm{~m}^{-1}$, $m$ being the fermionic mass. Taking for $m$ an average of the masses of the quarks composing the proton, we obtain a "confining" length $L_{p}^{(c)}$ which is comparable with an estimated proton diameter.
\end{abstract}

PACS number(s): 11.10.Jj; 11.10.Kk; 11.15.Pg

\section{INTRODUCTION}

The currently accepted theory of strong interactions, Quantum Chromodynamics (QCD), has an intricate structure which makes it difficult to be directly handled. For this reason, simplified models have been largely employed over the last decades in trials to obtain clues about the behavior of interacting quarks. Among them one of the most celebrated is the Gross-Neveu model [1], which considers the direct four-fermions interaction. In this paper, we consider the $N$-component tridimensional massive Gross-Neveu model, in the large- $N$ limit, compactified along one of the spatial directions. From a physical point of view, the model is intended to describe fermions bounded between two parallel planes, a distance $L$ apart from one another. We will call from now on quarks the fermions associated to the fermionic field, being understood that we are dealing with the quanta resulting from the quantization of the Gross-Neveu model. We define from the four-point function, an effective coupling constant $g(3, L ; \lambda)$ in the large- $N$ limit, which presents different behaviours if the fixed coupling constant $\lambda$ in unbounded space is below or above some "critical" value $\lambda_{c}$. For $\lambda<\lambda_{c}$ (weak coupling) the model presents smalldistance asymptotic freedom, $g(3, L ; \lambda) \rightarrow 0$, as $L \rightarrow 0$. For strong coupling $\left(\lambda>\lambda_{c}\right)$, starting from small values of $L$, we analytically demonstrate the simultaneous existence of short distance asymptotic freedom and of a singularity for $L$ equal to some $L=L^{(c)}, g(3, L ; \lambda) \rightarrow \infty$, as $L \rightarrow L_{-}^{(c)}$. This means that in the strong coupling

\footnotetext{
*Permanent adress : CBPF/MCT, Rua Dr. X. Sigaud 150, 22290-
} 180, Rio de Janeiro, RJ, Brazil regime, if we start with a system of a quark-antiquark pair bounded between two planes a distance $L<L^{(c)}$, from one another it would not be possible to separate them a distance larger than $L^{(c)}$. This spatial confinement of the quark-antiquark pair could be interpreted as the existence of bound states, characteristic of the model in the strong coupling regime. We will refer later to this property of the model as confinement, understood as the spatial confinement described above, not of colour confinement as it should happpens for QCD. An interesting point is that the confining length obtained is comparable with an estimated classical proton diameter.

Although perturbatively non renormalizable for dimensions $D>2$, the massive Gross-Neveu model in tridimensional space has been shown to exist and has been constructed in Ref. 2]. The idea that perturbative renormalizability should not be an absolute criterion to a model to be physically consistent comes from works done more than one decade ago. The results in renormalization theory reported in Refs. 3, 4] give a theoretical foundation to support the idea that non perturbatively renormalizable models can exist and have a physical meaning [5]. In fact, the Gross-Neveu model for dimensions greater than $D=2$ has been investigated since the middle of the seventies. Since then we know that the difficulties related to the perturbative non-renormalizability of the model can be surmounted, at least for $D=3$, by performing partial summations in the bare perturbation series, while maintaining its initial structure (see Refs. [1, [6, 7]). This point has been also studied in Ref. [8]. The essence of the method lies in the fact that the model in three dimensions may acquire a naive power counting behavior of a just renormalizable theory, if we perform a partial summation, to all orders in the coupling constant, of the chains of one-loop bubbles before trying to 
renormalize the perturbation series directly.

In present work, we perform such a summation for the large- $N$ model and we renormalize it employing a modified minimal subtraction scheme, where the coupling constant counter-term is a pole at the physical value of the argument of generalized Epstein zeta-functions [9]. This technique, which employs analytic and zeta-function regularizations, has been recently used to study the boundary dependence of the coupling constant and the spontaneous symmetry breaking in the compactified $\phi^{4}$ model [10, 11].

\section{THE COMPACTIFIED MODEL}

We start from the Wick-ordered massive Gross-Neveu Lagrangian in Euclidian metrics (in relativistic units, $\hbar=$ $c=1)$,

$$
\mathcal{L}=: \bar{\psi}(x)\left(i \gamma^{\mu} \partial_{\mu}+m\right) \psi(x):+\frac{u}{2}:[\bar{\psi}(x) \psi(x)]^{2}:,
$$

where $m$ is the mass, $u$ the coupling constant and $x$ is a point of $\mathbf{R}^{D}$. The fermionic field of spin $\frac{1}{2}$ has $N$ components, $\psi^{a}(x) a=1,2, \ldots, N$, and summation over flavor and spin indices are understood. For a finite number of flavors $N$, for $2<D<4$, a throughout study has been done in Ref. [12]. Here we consider the limit $N \rightarrow \infty$, which permits considerable simplifications.

In the language of Ref. 2] a theorem has been proved stating that a family of parameters can be exhibited such that, for $N$ sufficiently large, all renormalized Schwinger functions of the type $S_{2 p}(m, \lambda, N), \mathrm{p}=1,2, \ldots$ exist. Thus we examine in the following the large- $N$ zero externalmomenta four-point function, which is the basic object for our definition of the renormalized coupling constant. The four-point function at leading order in $\frac{1}{N}$ is given by the sum of all chains of single one-loop diagrams. This sum gives for the four-point function at zero external momenta the formal expression 2]

$$
\Gamma_{D}^{(4)}(0)=\frac{u}{1+N u \Sigma(D)},
$$

where $\Sigma(D)$ is the Feynman integral corresponding to the single one-loop four-point subdiagram

$$
\Sigma(D)=\int \frac{d^{D} k}{(2 \pi)^{D}} \frac{m^{2}-k^{2}}{\left(k^{2}+m^{2}\right)^{2}} .
$$

Notice that $\Sigma(D)$ has dimension of ass $^{D-2}$ and the coupling constant $u$ has dimension of mass $^{2-D}$.

We consider the system bounded between two parallel planes, normal to the $x$-axis, a distance $L$ apart from one another. We use Cartesian coordinates $\mathbf{r}=(x, \mathbf{z})$, where $\mathbf{z}$ is a $(D-1)$-dimensional vector, with corresponding momenta $\mathbf{k}=\left(k_{x}, \mathbf{q}\right)$, $\mathbf{q}$ being a $(D-1)$-dimensional vector in momenta space. In addition, we assume that the field satisfies bag-model boundary conditions [13, 14] on the planes $x=0$ and $x=L$. These boundary conditions, constraining the $x$-dependence of the field to a segment of length $L$, allow us to proceed with respect to the $x$ -coordinate in a manner analogous as it is done in the imaginary-time formalism in field theory. This extension of the Matsubara procedure to describe a model compactified in a space-coordinate has been already used in the case of bosonic fields [10, 11]. Accordingly, for fermionic fields, the Feynman rules should be modified following the prescription

$$
\int \frac{d k_{x}}{2 \pi} \rightarrow \frac{1}{L} \sum_{n=-\infty}^{+\infty}, \quad k_{x} \rightarrow \frac{2\left(n+\frac{1}{2}\right) \pi}{L} \equiv \omega_{n} .
$$

Then, the $L$-dependent four-point function at zero external momenta has the formal expression,

$$
\Gamma_{D}^{(4)}(0, L)=\frac{u}{1+N u \Sigma(D, L)},
$$

where $\Sigma(D, L)$ is the $L$-dependent Feynman sum-integral corresponding to the single one-loop subdiagram,

$$
\Sigma(D, L)=\frac{1}{L} \sum_{n=-\infty}^{\infty} \int \frac{d^{D-1} k}{(2 \pi)^{D-1}} \frac{m^{2}-\left(\mathbf{k}^{2}+\omega_{n}^{2}\right)}{\left(\mathbf{k}^{2}+\omega_{n}^{2}+m^{2}\right)^{2}} .
$$

Using the dimensionless quantities $q_{j}=k_{j} / 2 \pi m(j=$ $1,2, \ldots, D-1)$ and $a=(m L)^{-2}$, the single bubble $\Sigma(D, L)$ can be written as

$$
\begin{aligned}
\Sigma(D, a)= & \left.\Sigma(D, a, s)\right|_{s=2} \\
= & m^{D-2(s-1)} \sqrt{a} \\
& \times\left.\left[\frac{1}{4 \pi^{2}} U_{1}(s, a)-U_{2}(s, a)-a U_{3}(s, a)\right]\right|_{s=2},
\end{aligned}
$$

where

$$
\begin{aligned}
U_{1}(s, a)= & \frac{\pi^{(D-1) / 2-2 s+2}}{2^{2 s-2}} \frac{\Gamma(s-(D-1) / 2)}{\Gamma(s)} \\
& \times \sum_{n=-\infty}^{+\infty}\left[a\left(n+\frac{1}{2}\right)^{2}+(2 \pi)^{-2}\right]^{(D-1) / 2-s}
\end{aligned}
$$

and

$$
\begin{aligned}
& U_{2}(s, a)=\frac{D-1}{2(s-1)} U_{1}(s-1, a), \\
& U_{3}(s, a)=\frac{1}{1-s} \frac{\partial}{\partial a} U_{1}(s-1, a) .
\end{aligned}
$$

These expressions were obtained by performing the integrals over $q_{j}$ in Eq. (6) using well-known dimensional regularization formulas [15].

Sums of the type appearing in Eq. (8) can be casted in the form

$$
\sum_{n=-\infty}^{+\infty}\left[a\left(n+\frac{1}{2}\right)^{2}+c^{2}\right]^{-\nu}=4^{\nu} Z_{1}^{4 c^{2}}(\nu, a)-Z_{1}^{c^{2}}(\nu, a),
$$


where

$$
Z_{1}^{b^{2}}(\nu, a)=\sum_{n=-\infty}^{+\infty}\left[a n^{2}+b^{2}\right]^{-\nu}
$$

is one of the generalized Epstein zeta-functions 9] defined for $\operatorname{Re} \nu>1 / 2$, which possesses the following analytic extension

$$
\begin{aligned}
Z_{1}^{b^{2}}(\nu, a)= & \frac{\sqrt{\pi}}{\sqrt{a} \Gamma(\nu)}\left[\frac{\Gamma(\nu-1 / 2)}{b^{2 \nu-1}}\right. \\
& \left.+4 \sum_{n=1}^{\infty}\left(\frac{b \sqrt{a}}{\pi n}\right)^{\frac{1}{2}-\nu} K_{\frac{1}{2}-\nu}\left(\frac{2 \pi b n}{\sqrt{a}}\right)\right]
\end{aligned}
$$

valid for the whole complex $\nu$-plane. Therefore, the functions $U_{l}(s, a)(l=1,2,3)$ can be also extended to the whole complex $s$-plane, and we obtain

$$
\begin{aligned}
U_{1}(s, a)= & \frac{h(s, D)}{\sqrt{a}}\left[\Gamma\left(s-\frac{D}{2}\right)+4 W(s, a)\right], \\
U_{2}(s, a)= & \frac{(D-1) h(s-1, D)}{2(s-1) \sqrt{a}} \\
& \times\left[\Gamma\left(s-1-\frac{D}{2}\right)+4 W(s-1, a)\right], \\
U_{3}(s, a)= & \frac{h(s-1, D)}{2(s-1) a \sqrt{a}}\left[\Gamma\left(s-1-\frac{D}{2}\right)\right. \\
& \left.+4 W(s-1, a)-8 a \frac{\partial}{\partial a} W(s-1, a)\right],
\end{aligned}
$$

where

$$
h(s, D)=\frac{\pi^{2-D / 2}}{2^{D-2} \Gamma(s)}
$$

and

$$
\begin{aligned}
W(s, a)= & 2 \sum_{n=1}^{\infty}\left(\frac{\sqrt{a}}{n}\right)^{\frac{D}{2}-s} K_{\frac{D}{2}-s}\left(\frac{2 n}{\sqrt{a}}\right) \\
& -\sum_{n=1}^{\infty}\left(\frac{2 \sqrt{a}}{n}\right)^{\frac{D}{2}-s} K_{\frac{D}{2}-s}\left(\frac{n}{\sqrt{a}}\right) .
\end{aligned}
$$

Replacing these expressions in Eq.(7), we see that $\Sigma(D, L, s)$ can be written in the form

$$
\Sigma(D, L, s)=H(D . s)+G(D, L, s),
$$

where $H(D, s)$ is a term independent of $a$, coming from the first parcels between the brackets in Eqs. (14), (15) and (16), while $G(D, L, s)$ is the term arising from the sum over the parcels containing the $W$-functions in these equations. We are using a modified minimal subtraction scheme, where the coupling constant counter-terms are poles appearing at the physical value $s=2$. Then the $L$-dependent correction to the coupling constant is proportional to the regular part of the analytical extension of the Epstein zeta -function in the neighborhood of the pole at $s=2$. We see from Eqs. (15) and (16) that, for $s=2$ and even dimensions $D \geq 2, H(D)$ is divergent due to the pole of the $\Gamma$-function. Accordingly this term should be subtracted to give the renormalized single bubble function $\Sigma_{R}(D, L)$. For sake of uniformity, the term $H(D)$ is also subtracted in the case of other dimensions $D$, where no poles of $\Gamma$-functions are present; in these cases, we perform a finite renormalization. Then, we simply get, for any dimension $D$,

$$
\begin{aligned}
\Sigma_{R}(D, L)= & m^{D-2(s-1)\left[\frac{h(s, D)}{\pi^{2}} W(s, a)\right.} \\
& -\frac{2 D h(s-1, D)}{s-1} W(s-1, a) \\
& \left.+\frac{4 h(s-1, D) a}{s-1} \frac{\partial}{\partial a} W(s-1, a)\right]\left.\right|_{s=2} .
\end{aligned}
$$

In dimension $D=3$, using the explicit form for the Bessel functions $K_{ \pm 1 / 2}(z)=\sqrt{\pi} e^{-z} / \sqrt{2 z}$ and performing the resulting sums, we obtain (remembering that $\left.a=(m L)^{-2}\right)$

$$
\begin{aligned}
\Sigma_{R}(3, L)= & m\left[\frac{4 \pi^{2}+1}{2 \pi}\left(\frac{e^{-2 m L}}{1-e^{-2 m L}}\right)\right. \\
& -\frac{4 \pi^{2}+1}{4 \pi}\left(\frac{e^{-m L}}{1-e^{-m L}}\right) \\
& \left.+\frac{2 \pi}{m L} \log \left(\frac{1-e^{-2 m L}}{1-e^{-m L}}\right)\right] .
\end{aligned}
$$

Substituting this expression into Eq. (5), we obtain the large- $N$ effective renormalized coupling constant, taking $N u=\lambda$ fixed as usual,

$$
g(3, L)=N \Gamma_{3 R}^{(4)}(0, L)=\frac{\lambda}{1+\lambda \Sigma_{R}(3, L)} .
$$

\section{BOUNDARY BEHAVIOUR}

Fig. 1 shows the renormalized single bubble (in units of $m$ ), $S=\Sigma_{R}(3, L) / m$, as a function of $L$ (in units of $m^{-1}$ ). We see from this plot and from Eq. (21) that $\lim _{L \rightarrow \infty} \Sigma_{R}(3, L)=0$ and that $\Sigma_{R}(3, L)$ diverges as $L \rightarrow 0$. This means that (see Eq. (22) the fixed coupling constant $\lambda$ corresponds to the large- $N$ renormalized effective coupling in absence of boundaries and that, independently of the value of $\lambda$, the system presents ultra-violet asymptotic freedom. Moreover, $\Sigma_{R}(3, L)$ has a zero at $L=L_{\text {min }}^{(c)} \simeq 2.07$, for which $g(3, L)=\lambda$.

The interesting point is to investigate what happens for finite values of $L$, when we start from $L$ near zero (in the region of asymptotic freedom). In Fig. 2, we have plotted the relative coupling constant $y=g(3, L) / \lambda$ as 


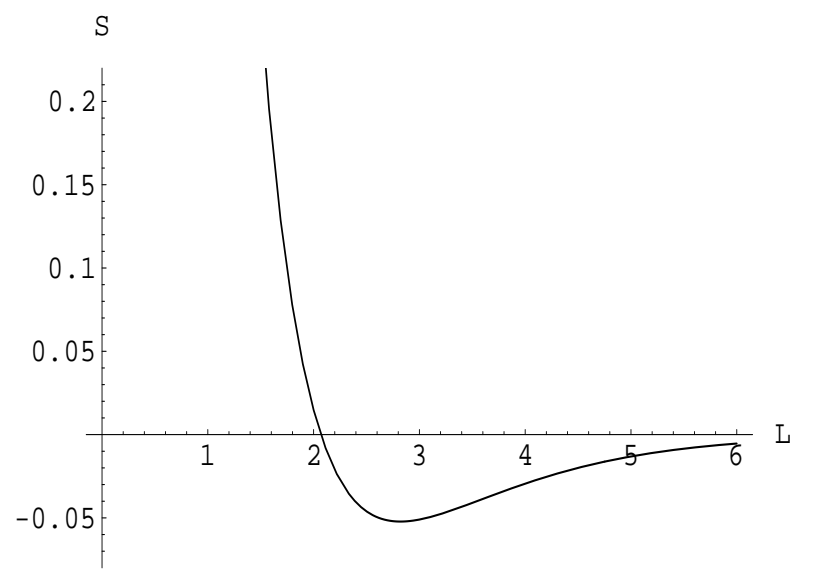

FIG. 1: Renormalized single bubble (in units of $m$ ), $S=$ $\Sigma_{R}(3, L) / m$, as a function of the length $L$ (in units of $m^{-1}$ ).

a function of $L$ (in units of $m^{-1}$ ), taking four different values of $\lambda$ (also in units of $m^{-1}$ ). For the smallest value considered $(\lambda=0.2)$, we only see asymptotic freedom with $g(3, L)$ varying from 0 (for $L=0$ ) up to $\lambda$ (for $L=$ $\infty$ ). Increasing the value of $\lambda$ (e.g. $\lambda=10$ and $\lambda=15$ in Fig. 2), all the curves present a peak at $L=L_{\max }^{(c)} \simeq 2.82$, the point at which $\lambda / g(3, L)$ reaches its minimum value. This minimum value of $\lambda / g(3, L)$ vanishes for $\lambda=\lambda_{c} \simeq$ 19.16 (corresponding to the full line in Fig. 2) and so the effective coupling constant $g\left(3, L_{\max }^{(c)}\right)$ diverges. This means that, for this value of $\lambda$, the system is absolutely confined in the slab of thickness $L_{\max }^{(c)}$. Taking greater values of $\lambda>\lambda_{c}$, the minimum of $\lambda / g(3, L)$ becomes negative, $g(3, L)$ goes to $\infty$ at a value $L^{(c)}<L_{\max }^{(c)}$ and the system would be confined in a thinner slab. However, as $\lambda$ goes to $\infty, L^{(c)}$ tends to a lower bound equal to $L_{\text {min }}^{(c)}$. In other words, for $\lambda_{c} \leq \lambda<\infty$, the effective coupling constant is singular for a critical value of $L$, namely $L^{(c)}(\lambda)$; the system is then confined in a slab of thickness $L^{(c)}(\lambda)$ lying in the interval $\left(L_{\min }^{(c)}, L_{\max }^{(c)}\right]$.

Searching for a physical interpretation of our results, we shall take the fixed coupling constant $\lambda=\lambda_{c}$ implying that the physical confining length is $L^{(c)}=L_{\max }^{(c)}$, the maximum conceived confining length. In order to estimate the value of $L^{(c)}$, we need to fix a value of $m$. We choose $m=3.25 \times 10^{11} \mathrm{~cm}^{-1}(\sim 6.5 \mathrm{Mev})$, corresponding to an average between typical $u$ - and $d$ quark masses (recall that the quark content of the proton is $\{u u d\})$ [16]. This choice gives a confining length $L_{p}^{(c)} \simeq 8.67 \times 10^{-12} \mathrm{~cm}$. This value should be compared with an estimated classical proton diameter, $2 R_{p} \sim$ $6.92 \times 10^{-12} \mathrm{~cm}\left[17\right.$; notice that this value is about $10^{2}$ times larger than the experimentally measured proton charge radius [18.

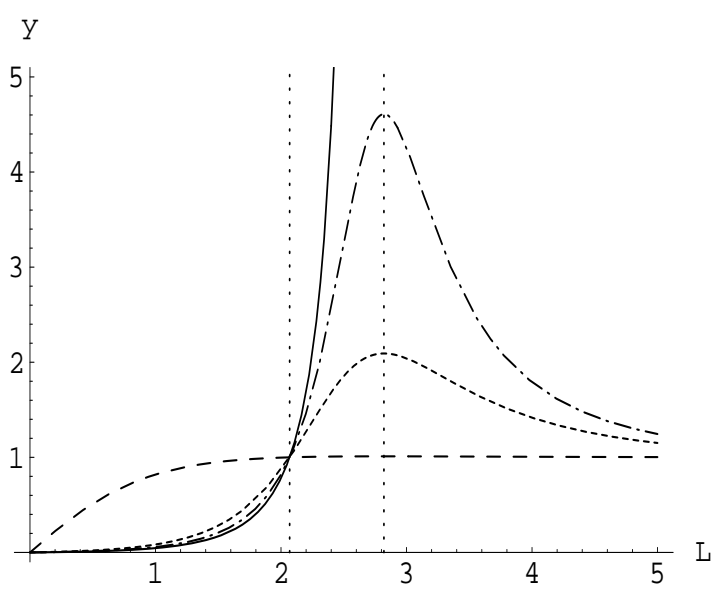

FIG. 2: Plots of the large- $N$ relative effective coupling constant, $y=g(3, L) / \lambda$, as a function of $L$ (in units of $m^{-1}$ ) for four different values of the fixed coupling constant $\lambda$ (in units of $\left.m^{-1}\right): \lambda=0.2$ (dashed line), $\lambda=10$ (dotted line), $\lambda=15$ (dotted-dashed line) and $\lambda=19.16$ (full line). The dotted vertical lines, passing by $L_{\min }^{(c)} \simeq 2.07$ and $L_{\max }^{(c)} \simeq 2.82$, are plotted as a visual guide.

\section{CONCLUDING REMARKS}

Under the assumption that the large- $N$ Gross-Neveu model can be thought about as a model sharing some of the properties of physically meaningful theories (in the sense of experimentally testable), we can say that we have exhibited in a direct way the properties of ultraviolet asymptotic freedom and confinement, characteristics of strong interactions. This could be contrasted with the fact that in $Q C D$, although asymptotic freedom is a well established property on theoretical grounds, confinement has been confirmed only by computer simulations in lattice field theory. Confinement has never been, to our knowledge, analytically proved.

It is worth mentioning that, in Ref. [10], we have analyzed the dependence of the coupling constant on $L$ for the large- $N \phi^{4}$ model, the bosonic counterpart of the Gross-Neveu model studied here. In that case of bosons, no confinement is found at all, but only asymptotic freedom is encountered. Comparison of the results derived in Ref. 10] with those of the present work suggests that the effect of confinement in hadronic matter may be mainly due to the fermionic nature of the quarks.

Finally, we would like to point out that the behavior described in this work should not be heavily dependent on the confining geometry. One expects to have ultraviolet asymptotic freedom in any case and partial or absolute confinement, depending on the value of $\lambda$. A thorough study on the subject with different geometries, and including temperature, is in progress and will be presented elsewhere. 


\section{ACKNOWLEDGEMENTS}

This work was supported by CAPES and CNPq, Brazilian Agencies. One of us (APCM) is grateful for the kind hospitality of the Centre de Physique Théorique,
Ecole Polytechnique and Instituto de Física, Universidade Federal da Bahia, where part of this work has been done. A.P.C.M. also thanks C. de Calan and J. Magnen from Ecole Polytechnique for interesting discussions.
[1] D. J. Gross and A. Neveu, Phys. Rev. D 10, 4410 (1974).

[2] C. de Calan, P. A. Faria da Veiga, J. Magnen, and R. Séneor, Phys. Rev. Lett. 66, 3233 (1991).

[3] K. Gawedzki and A. Kupiainen, Phys. Rev. Lett. 55, 363 (1985).

[4] K. Gawedzki and A. Kupianen, Nucl. Phys. B 262, 33 (1985).

[5] S. Weinberg, Phys. Rev. D 56, 2303 (1997).

[6] G. Parisi, Nucl. Phys. B 100, 368 (1975).

[7] Y. Arefv́a, E. Nissimov, and S. Pacheva, Comm. Math. Phys. 71, 213 (1980).

[8] B. Rosenstein, B. J. Warr, and S. H. Park, Phys. Rev. Lett. 62, 1433 (1989).

[9] P. Epstein, Math. Ann. 56, 615 (1903), 67, 205 (1907); A. Elizalde and E. Romeo, J. Math. Phys. 30, 1133 (1989); K. Kirsten, J. Math. Phys. 35, 459 (1994).

[10] A. P. C. Malbouisson and J. M. C. Malbouisson, J. Phys. A: Math. Gen. 35, 2263 (2002).
[11] A. P. C. Malbouisson, J. M. C. Malbouisson, and A. E. Santana, Nucl. Phys. B 631, 83 (2002).

[12] S. Hands, A. Kocić, and J. B. Kogut, Ann. Phys. 224, 29 (1993).

[13] A. Chodos, R. L. Jaffe, K. Johnson, C. B. Thorn, and V. F. Weisskopf, Phys. Rev. D 9, 3471 (1974).

[14] C. A. Lütken and F. Ravndal, J. Phys. A: Math. Gen. 21, L793 (1988).

[15] P. Ramond, Field Theory: a modern primer (AddisonWesley, Redwood, 1990), Appendix B.

[16] K. Hagiwara et al. Particle Data Group, Phys. Rev. D 66, 010001 (2002).

[17] This estimate was obtained from the proton-electron mass ratio and from the tabulated classical electron radius, assuming equal densities.

[18] S. G. Karshenboim, Can. J. Phys. 77, 241 (1999). 